\title{
A Flexible Biphenyl Chromophore as CD Probe for the Assignment of the Absolute Configuration of Carboxylic Acids
}

\author{
Stefano Superchi, Roberto Bisaccia, Daniele Casarini, Alessandro Laurita and Carlo Rosini* \\ Dipartimento di Chimica, Università della Basilicata, via N. Sauro 85, 85100, Potenza, Italy.
}

\section{Supplementary Material}

Full characterization and experimental procedures for compounds $\mathbf{2 a}-\mathbf{m}$. VT-NMR experiments for

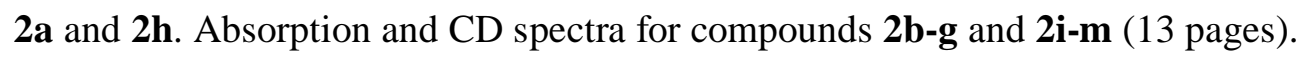

General Procedures. Melting points are uncorrected. Optical rotations were measured at r.t. on a Jasco DIP-370 polarimeter. ${ }^{1} \mathrm{H}$ and ${ }^{13} \mathrm{C}$ NMR spectra were recorded on a Bruker Aspect 300 or a Varian INOVA 600 spectrometer. Analytical TLC were performed on $0.20 \mathrm{~mm}$ silica gel plates Macherey-Nagel ALUGRAM SIL G/UV 254 and column chromatography was carried out with silica gel Macherey-Nagel 60 (70-230 mesh). Absorption and CD spectra of compounds 2a-m were recorded on a JASCO J600 spectropolarimeter at room temperature, in freshly distilled THF, using $0.1 \mathrm{~mm}$ cells and concentrations of about $1 \times 10^{-3} \mathrm{M}$. During the measurement, the instrument was thoroughly purged with nitrogen. Toluene and THF were freshly distilled prior their use on sodium benzophenone ketyl under nitrogen atmosphere. $\mathrm{CH}_{2} \mathrm{Cl}_{2}$ and triethylamine were distilled over $\mathrm{CaH}$ prior their use and stored under nitrogen atmosphere. (S)-(-)-2-bromo-propionic acid (1a), $(R)-(-)-5-$ oxo-2-tetrahydrofuran carboxylic acid (1c), l'(R)-(+)-3,3,3-trifluoro-2-metoxy-2-phenyl-propionic acid (1j), L-3-phenyllactic acid (1m) (Aldrich); L-lactic acid (1e), (R)-(-)-2-phenylpropionic acid (1h) (Lancaster); (S)-(+)-2-metylbutirric acid (1b), (S)-(+)-hexahydromandelic acid (1d), Lphenylglycine-N-Boc (1k), (S)-(+)-ibuprofen (1i) (Fluka) are commercially available and were used as purchased. Preparation of the biphenyl amine $\mathbf{3}$ was carried out as described elsewhere. ${ }^{1}$

\section{General procedure for the synthesis of biphenyl amides}

To a solution of the biphenyl amine $3(60 \mathrm{mg}, 0.31 \mathrm{mmol})$ in anhydrous $\mathrm{CH}_{2} \mathrm{Cl}_{2}(3 \mathrm{~mL})$ were added, in sequence, the optically active acid (0.25 mmol), 1-[3-(Dimethylamino)propyl]-3ethylcarbodiimide chloride (EDC) $(79.6 \mathrm{mg}, 0.4153 \mathrm{mmol})$, and DMAP (30.4mg, $0.25 \mathrm{mmol})$. The resulting solution was stirred overnight at r.t., then diluted with $\mathrm{CH}_{2} \mathrm{Cl}_{2}(5 \mathrm{~mL})$, washed with $10 \%$ aqueous $\mathrm{NaHCO}_{3}(3 \times 10 \mathrm{~mL})$, brine $(2 \times 10 \mathrm{~mL})$, and dried over anhydrous $\mathrm{Na}_{2} \mathrm{SO}_{4}$. After

\footnotetext{
${ }^{1}$ Scafato, P.; Cunsolo, G.; Labano, S., Rosini, C. Tetrahedron 2004, 60, 8801.
} 
evaporation of solvent the recovered residue was purified by column chromatography $\left(\mathrm{CH}_{2} \mathrm{Cl}_{2} / \mathrm{MeOH}\right)(97 / 3)$ affording the corresponding biphenyl amide in 50-80\%yield.

6,7-Dihydro-5H-dibenzo[c,e]azepine (S)-2-bromopropanoyl amide (2a): Colourless prisms; ${ }^{1} \mathrm{H}$ NMR $\left(600 \mathrm{MHz}, \mathrm{CDCl}_{3}\right) \delta 1.95(\mathrm{~d}, J=6.5 \mathrm{~Hz}, 3 \mathrm{H}, \mathrm{Me}), 4.14\left(\mathrm{~d}, J=13 \mathrm{~Hz}, 2 \mathrm{H}, \mathrm{NCH}_{2}\right), 4.56(\mathrm{~d}, J$ $\left.=13 \mathrm{~Hz}, 1 \mathrm{H}, \mathrm{NCH}_{2}\right), 4.70(\mathrm{q}, J=6.5 \mathrm{~Hz}, 1 \mathrm{H}, \mathrm{CH}-\mathrm{Me}), 4.76\left(\mathrm{br} \mathrm{d}, 1 \mathrm{H}, \mathrm{NCH}_{2}\right), 7.38-7.42(\mathrm{~m}, 2 \mathrm{H}$, Ar), $7.44(\mathrm{~d}, 1 \mathrm{H}, \mathrm{Ar}), 7.47-7.58(\mathrm{~m}, 5 \mathrm{H}, \mathrm{Ar}) ;{ }^{13} \mathrm{C} \mathrm{NMR}\left(150 \mathrm{MHz}, \mathrm{CDCl}_{3}\right) \delta 21.70\left(\mathrm{CH}_{3}\right)$, 38.98(CH-Me), 46.74( $\left(\mathrm{NCH}_{2}\right), 49.05\left(\mathrm{NCH}_{2}\right), 127.92(\mathrm{CH}), 128.16(\mathrm{CH}), 128.30(\mathrm{CH}), 128.42(\mathrm{CH})$, 128.78(CH), 129.06(CH), 129.67(CH), 130.20(CH), 132.75(quat), 133.43(quat), 140.40(quat), 140.65(quat), 166.75(NCO). Anal. Calcd for $\mathrm{C}_{17} \mathrm{H}_{16} \mathrm{BrNO}$ : C, 61.83; H, 4.88; N, 4.24. Found: C, 61.57; H, 4.92; N, 4.33.

6,7-Dihydro-5H-dibenzo[c,e]azepine $(S)-2$-methylbutanoyl amide $(2 \mathrm{~b})$ : Clear oil; $[\alpha]_{\mathrm{D}}=-4.0(c$ $\left.=0.95, \mathrm{CHCl}_{3}\right) ;{ }^{1} \mathrm{H} \mathrm{NMR}\left(300 \mathrm{MHz}, \mathrm{CDCl}_{3}\right) \delta 0.95(\mathrm{t}, J=7.5 \mathrm{~Hz}, 3 \mathrm{H}), 1.21(\mathrm{~d}, J=6 \mathrm{~Hz}, 3 \mathrm{H})$, $1.55(\mathrm{~m}, 1 \mathrm{H}), 1.85(\mathrm{~m}, 1 \mathrm{H}), 2.80(\mathrm{~m}, 1 \mathrm{H}), 4.31(\mathrm{~s}, 2 \mathrm{H}), 4.38(\mathrm{~d}, J=13.3 \mathrm{~Hz}, 1 \mathrm{H}), 4.5(\mathrm{~d}, J=13.3$ $\mathrm{Hz}, 1 \mathrm{H}), 7.3-7.6(\mathrm{~m}, 8 \mathrm{H}) ;{ }^{13} \mathrm{C} \mathrm{NMR}\left(75.5 \mathrm{MHz}, \mathrm{CDCl}_{3}\right) \delta 11.93,17.29,27.24,37.95,46.31,49.07$, 127.84, 128.21, 128.39, 128.48, 128.87, 129.08, 133.77, 134.49, 140.50, 140.87, 174.52. Anal. Calcd for $\mathrm{C}_{19} \mathrm{H}_{21} \mathrm{NO}$ : C, 81.68; H, 7.58; N, 5.01. Found: C, 81.75; H, 7.63; N, 4.95.

6,7-Dihydro-5H-dibenzo[c,e]azepine $(R)$-5-oxo-2-tetrahydrofuroyl amide (2c): Pale yellow prisms; m.p. $54.2-55.0^{\circ} \mathrm{C} .[\alpha]_{\mathrm{D}}=-37.8\left(c=0.86, \mathrm{CHCl}_{3}\right) ;{ }^{1} \mathrm{H} \mathrm{NMR}\left(300 \mathrm{MHz}, \mathrm{CDCl}_{3}\right) \delta 2.45(\mathrm{~m}$, $1 \mathrm{H}), 2.55(\mathrm{~m}, 1 \mathrm{H}), 2.80(\mathrm{~m}, 2 \mathrm{H}), 4.2(\mathrm{~d}, J=12.0 \mathrm{~Hz}, 1 \mathrm{H}), 4.35(\mathrm{~d}, J=12.0 \mathrm{~Hz}, 1 \mathrm{H}), 4.48(\mathrm{~d}, J=$ $12.0 \mathrm{~Hz}, 1 \mathrm{H}), 5.37\left(\mathrm{dd}, J^{\prime}=6.6 \mathrm{~Hz}, J^{\prime}{ }^{\prime}=3.3 \mathrm{~Hz}, 1 \mathrm{H}\right), 7.4-7.7(\mathrm{~m}, 8 \mathrm{H}) ;{ }^{13} \mathrm{C} \mathrm{NMR}(75.5 \mathrm{MHz}$, $\left.\mathrm{CDCl}_{3}\right) \delta 24.67,27.75,47.38,48.94,75.81,128.44,128.54,128.78,128.79,129.24,129.50$, $129.59,130.56,132.99,133.352,140.76,140.85,165.64,176.55$. Anal. Calcd for $\mathrm{C}_{19} \mathrm{H}_{17} \mathrm{NO}_{3}: \mathrm{C}$, 74.25; H, 5.58; N, 4.56. Found: C, 74.05; H, 5.45; N, 4.62.

6,7-Dihydro-5H-dibenzo[c,e]azepine $(S)$-2-ciclohexyl-2-hydroxyacetyl amide (2d): Pale yellow prisms; m.p. $58.3-60.5^{\circ} \mathrm{C} ;[\alpha]_{\mathrm{D}}=-84.8\left(c=1.06, \mathrm{CHCl}_{3}\right) ;{ }^{1} \mathrm{H} \mathrm{NMR}\left(300 \mathrm{MHz}, \mathrm{CDCl}_{3}\right) \delta 1.0-1.03$ $(\mathrm{m}, 5 \mathrm{H}), 1.3-1.8(\mathrm{~m}, 6 \mathrm{H}), 3.6(\mathrm{~d}, J=7.5,1 \mathrm{H}), 4.04(\mathrm{~d}, J=13.3 \mathrm{~Hz}, 1 \mathrm{H}), 4.2(\mathrm{~d}, J=13.3 \mathrm{~Hz}, 1 \mathrm{H})$, $4.26(\mathrm{~d}, J=15 \mathrm{~Hz}, 1 \mathrm{H}), 4.35\left(\mathrm{dd}, J^{\prime}=6.5 \mathrm{~Hz}, J^{\prime \prime}=0.6 \mathrm{~Hz}, 1 \mathrm{H}\right), 4.52$ ( d, $\left.J=15 \mathrm{~Hz}, 1 \mathrm{H}\right),(7.2-7.6$ $(\mathrm{m}, 8 \mathrm{H}) ;{ }^{13} \mathrm{C} \mathrm{NMR}\left(75.5 \mathrm{MHz}, \mathrm{CDCl}_{3}\right) \delta 25.46,25.95,26.52,29.95,41.86,42.13,48.42,72.68$, $128.09,128.28,128.42,128.53,128.66,129.09,129.20,130.18,132.72,133.27,140.46,140.61$, 171.64. Anal. Calcd for $\mathrm{C}_{22} \mathrm{H}_{25} \mathrm{NO}_{2}$ : C, 78.77; H, 7.51; N, 4.18. Found: C, 78.57; H, 7.46; N, 4.15. 
6,7-Dihydro-5H-dibenzo[c,e]azepine (S)-2-hydroxypropanoyl amide (2e): Yellow prisms; m.p. $=68.2-70.0^{\circ} \mathrm{C} ;[\alpha]_{\mathrm{D}}=-121.4\left(c=1.0, \mathrm{CHCl}_{3}\right) ;{ }^{1} \mathrm{H} \mathrm{NMR}\left(600 \mathrm{MHz}, \mathrm{CDCl}_{3}\right) \delta 1.47(\mathrm{~d}, J=6.7 \mathrm{~Hz}$, $3 \mathrm{H}, \mathrm{Me}), 3.94(\mathrm{~d}, J=7.0 \mathrm{~Hz}, 1 \mathrm{H}, \mathrm{OH}), 4.14\left(\mathrm{~d}, J=13.2 \mathrm{~Hz}, 1 \mathrm{H}, \mathrm{NCH}_{2}\right), 4.28(\mathrm{~d}, J=13.2 \mathrm{~Hz}, 1 \mathrm{H}$, $\mathrm{NCH}_{2}$ ), 4.44 (br d, 1H, $\mathrm{NCH}_{2}$ ), 4.58 (br d, 1H, $\mathrm{NCH}_{2}$ ), 4.69 (m, J = 6.7 Hz, 1H, CH-Me), 7.38 (d,1H, Ar), 7.44 (t, 2H, Ar), $7.48(\mathrm{~d}, 1 \mathrm{H}, \mathrm{Ar}), 7.51-7.55$ (m, 2H, Ar), $7.56(\mathrm{~d}, 1 \mathrm{H}, \mathrm{Ar}), 7.59$ (d,1H, $\mathrm{Ar}) ;{ }^{13} \mathrm{C}$ NMR $\left(150 \mathrm{MHz}, \mathrm{CD}_{2} \mathrm{Cl}_{2}\right) \delta 21.36\left(\mathrm{CH}_{3}\right), 46.97\left(\mathrm{NCH}_{2}\right), 48.24\left(\mathrm{NCH}_{2}\right), 64.66(\mathrm{OCH})$, 128.12(CH, C6), 128.30(CH, C6'), $128.39(\mathrm{CH}, \mathrm{C} 4), 128.47(\mathrm{CH}, \mathrm{C} 4$ '), $128.85(\mathrm{CH}, \mathrm{C} 5)$, 129.14(CH, C5'), 129.20 (CH, C3), 130.18(CH, C3'), 132.89(quat), 133.39(quat), 140.56(quat), 140.73(quat), 173.08(NCO). Anal. Calcd for $\mathrm{C}_{17} \mathrm{H}_{17} \mathrm{NO}_{2}$ : C, 76.38; H, 6.41; N, 5.24. Found: C, $76.45 ; \mathrm{H}, 6.33 ; \mathrm{N}, 5.35$.

6,7-Dihydro-5H-dibenzo[c,e]azepine (S)- $N$-BOC-2-aminopropanoyl amide (2f): Clear oil; ${ }^{1} \mathrm{H}$ NMR $\left(300 \mathrm{MHz}, \mathrm{CDCl}_{3}\right) \delta 1.49(\mathrm{~m}, 12 \mathrm{H}), 4.2(\mathrm{~d}, J=11.25 \mathrm{~Hz}, 1 \mathrm{H}), 4.4(\mathrm{~d}, J=11.25 \mathrm{~Hz}, 3 \mathrm{H})$, 4.85 (quint, $J=6.0 \mathrm{~Hz}, 1 \mathrm{H}), 5.64(\mathrm{~d}, J=5.25 \mathrm{~Hz}, 1 \mathrm{H}), 7.35-7.6(\mathrm{~m}, 8 \mathrm{H}) ;{ }^{13} \mathrm{C} \mathrm{NMR}(75.5 \mathrm{MHz}$, $\left.\mathrm{CDCl}_{3}\right) \delta 19.54,28.42,29.06,46.69,46.61,48.8,76.57,76.99,77.41,79.56,128.06,128.22$, $128.42,128.47,128.81,129.10,129.35,130.21,133.05,133.61,140.72,170.64$. Anal. Calcd for $\mathrm{C}_{22} \mathrm{H}_{26} \mathrm{~N}_{2} \mathrm{O}_{3}: \mathrm{C}, 72.11 ; \mathrm{H}, 7.15 ; \mathrm{N}, 7.64$. Found: C, 72.23; H, 7.10; N, 7.70.

6,7-Dihydro-5H-dibenzo[c,e]azepine ( $S$ )- $N$-BOC-2-amino-3-methylbutanoyl amide (2g): Pale yellow prisms; m.p. 84.6-84.7 ${ }^{\circ} \mathrm{C} ;[\alpha]_{\mathrm{D}}=21.3\left(c=1.62, \mathrm{CHCl}_{3}\right) ;{ }^{1} \mathrm{H}$ NMR $\left(300 \mathrm{MHz}, \mathrm{CDCl}_{3}\right) \delta$ $0.95(\mathrm{~d}, J=3.7 \mathrm{~Hz}, 3 \mathrm{H}), 1.0(\mathrm{~d}, J=3.7 \mathrm{~Hz}, 3 \mathrm{H}), 1.49$ (s, 9H), 4.05 (t, $J=7.5 \mathrm{~Hz}, 1 \mathrm{H}), 4.2(\mathrm{~d}, J=$ $16.5 \mathrm{~Hz}, 2 \mathrm{H}), 4.65(\mathrm{~m}, 2 \mathrm{H}), 5.0(\mathrm{~d}, J=9 \mathrm{~Hz}, 1 \mathrm{H}), 5.4(\mathrm{~d}, J=9 \mathrm{~Hz}, 1 \mathrm{H}), 7.3-7.6(\mathrm{~m}, 7 \mathrm{H}) ;{ }^{13} \mathrm{C} \mathrm{NMR}$ $\left(75.5 \mathrm{MHz}, \mathrm{CDCl}_{3}\right) \delta 17.51,19.62,28.29,29.67,31.54,46.66,49.03,55.64,67.63,107.89,128.01$, $128.05,128.26,128.4,128.45,128.76,129.01,129.51,130.1,133.19,133.72,140.59,155.67$, 170.28. Anal. Calcd for $\mathrm{C}_{24} \mathrm{H}_{30} \mathrm{~N}_{2} \mathrm{O}_{3}$ : C, 73.07; H, 7.66; N, 7.10. Found: C, 73.15; H, 7.53; N, 7.18.

6,7-Dihydro-5H-dibenzo[c,e]azepine $(R)-2$-phenylpropanoyl amide (2h): White plates; m.p. = 174.5-177.0 ${ }^{\circ} \mathrm{C} ;[\alpha]_{\mathrm{D}}=-184\left(c=0.99, \mathrm{CHCl}_{3}\right) ;{ }^{1} \mathrm{H} \mathrm{NMR}\left(300 \mathrm{MHz}, \mathrm{CDCl}_{3}\right) \delta 1.49(\mathrm{~d}, J=6.9 \mathrm{~Hz}$, $3 \mathrm{H}), 3.97(\mathrm{~m}, 3 \mathrm{H}), 4.28(\mathrm{~d}, J=12 \mathrm{~Hz}, 1 \mathrm{H}), 4.9(\mathrm{~d}, J=12 \mathrm{~Hz}, 1 \mathrm{H}), 6.23(\mathrm{~d}, 7.36 \mathrm{~Hz}, 1 \mathrm{H}), 7.10(\mathrm{t}, J$ $=7.3 \mathrm{~Hz}, 1 \mathrm{H}), 7.2-7.6(\mathrm{~m}, 11 \mathrm{H}) ;{ }^{13} \mathrm{C} \mathrm{NMR}\left(150 \mathrm{MHz}, \mathrm{CDCl}_{3}\right) \delta 20.9\left(\mathrm{CH}_{3}\right), 44.4(\mathrm{CHMe})$, 46.5 $\left(\mathrm{NCH}_{2}\right), 48.2\left(\mathrm{NCH}_{2}\right), 126.84(\mathrm{CH}), 127.37(2 \mathrm{CH}, \mathrm{Ph}), 127.79(\mathrm{CH}), 127.81(\mathrm{CH}), 127.94(\mathrm{CH})$, 128.24(CH), 128.49(CH), 128.57(CH), 128.82(CH), 129.10(2CH, Ph), 130.19(CH), 133.22(quat), 133.95(quat), 140.52(quat), 140.55 (quat), 142.40 (quat), 171.18(NCO). Anal. Calcd for $\mathrm{C}_{23} \mathrm{H}_{21} \mathrm{NO}$ : C, 84.37; H, 6.46; N, 4.28. Found: C, 84.45; H, 6.39; N, 4.35. 
6,7-Dihydro-5H-dibenzo[c,e]azepine (S)-2-(4-isobutylphenyl)-propanoyl amide (2i): Yellow cubic prisms; m.p. $=144.6-145.3^{\circ} \mathrm{C} ;[\alpha]_{\mathrm{D}}=+171.71\left(c=1.05, \mathrm{CHCl}_{3}\right) ;{ }^{1} \mathrm{H} \mathrm{NMR}(600 \mathrm{MHz}$, $\left.\mathrm{CDCl}_{3}\right) \delta 1.52(\mathrm{~d}, J=6.8 \mathrm{~Hz}, 3 \mathrm{H}, \mathrm{Me}), 4.14\left(\mathrm{~d}, 2 \mathrm{H}, J=13.2 \mathrm{~Hz}, \mathrm{NCH}_{2}\right), 4.56(\mathrm{~d}, J=13.2 \mathrm{~Hz}, 1 \mathrm{H}$, $\mathrm{NCH}_{2}$ ), 4.70 (q, $\left.J=6.7 \mathrm{~Hz}, 1 \mathrm{H}, C H-\mathrm{Me}\right), 4.76$ (br d, 1H, $\left.\mathrm{NCH}_{2}\right), 6.28$ (br d, 1H, Ar), 7.13 (d t, $J$ $\left.=7.3 \mathrm{~Hz} ; J^{\prime \prime}=1.2 \mathrm{~Hz}, 1 \mathrm{H}, \mathrm{Ar}\right), 7.29-7.34(\mathrm{~m}, 3 \mathrm{H}, \mathrm{Ar}), 7.36-7.40(\mathrm{~m}, 4 \mathrm{H}, \mathrm{Ar}), 7.43-7.48$ (m, 4H, Ar). ${ }^{13} \mathrm{C}$ NMR $\left(150 \mathrm{MHz}, \mathrm{CDCl}_{3}\right) \delta 20.95\left(\mathrm{CH}_{3}\right), 44.43\left(\mathrm{NCH}_{2}\right), 46.55\left(\mathrm{NCH}_{2}\right), 48.23(\mathrm{CHMe})$, 126.84(CH), 127.37(2CH, Ph), 127.79(CH), 127.81(CH), 127.94(CH), 128.24(CH), 128.49(CH), 128.57(CH), 128.82(CH), 129.10(2CH,Ph), 130.19(CH, Ph), 133.22(quat), 133.95(quat), 140.52(quat), 140.55 (quat), 142.40(quat), 171.18(NCO). Anal. Calcd for $\mathrm{C}_{27} \mathrm{H}_{29} \mathrm{NO}$ : C, 84.55; $\mathrm{H}$, 7.62; N, 3.65. Found: C, 84.43; H, 7.69; N, 3.62 .

6,7-Dihydro-5H-dibenzo[c,e]azepine $(R)$-2-trifluorometyl-2-methoxy-2-phenylacetyl amide (2j): Yellow prisms; m.p. $=208.5-210.4{ }^{\circ} \mathrm{C} ;[\alpha]_{\mathrm{D}}=+132.81\left(c=0.83, \mathrm{CHCl}_{3}\right) ;{ }^{1} \mathrm{H}$ NMR $(300$ $\left.\mathrm{MHz} \mathrm{CDCl}_{3}\right) \delta 3.7(\mathrm{~s}, 3 \mathrm{H}), 3.8(\mathrm{~d}, J=13.6 \mathrm{~Hz}, 1 \mathrm{H}), 3.95(\mathrm{~d}, J=13.6 \mathrm{~Hz}, 1 \mathrm{H}), 4.35(\mathrm{~d}, J=13.6$ $\mathrm{Hz}, 1 \mathrm{H}), 5.0(\mathrm{~d}, J=13.6 \mathrm{~Hz}, 1 \mathrm{H}), 5.7(\mathrm{bs}, 1 \mathrm{H}), 7.05(\mathrm{t}, J=6.8 \mathrm{~Hz}, 1 \mathrm{H}), 7.3-7.6(\mathrm{~m}, 10 \mathrm{H}), 7.65(\mathrm{~d}$, $J=6.8 \mathrm{~Hz}, 2 \mathrm{H})$. Anal. Calcd for $\mathrm{C}_{24} \mathrm{H}_{20} \mathrm{~F}_{3} \mathrm{NO}_{2}: \mathrm{C}, 70.06 ; \mathrm{H}, 4.90 ; \mathrm{N}, 3.40$. Found: C, 70.12; H, $4.83 ; \mathrm{N}, 3.47$.

6,7-Dihydro-5H-dibenzo[c,e]azepine $\quad(S)-N$-BOC-2-amino-2-phenylacetyl amide (2k): Colourless prisms; m.p. $=48.3-50.4^{\circ} \mathrm{C} ;[\alpha]_{\mathrm{D}}=+112.04\left(c=0.93, \mathrm{CHCl}_{3}\right) ;{ }^{1} \mathrm{H}^{\mathrm{NMR}}(300 \mathrm{MHz}$, $\left.\mathrm{CDCl}_{3}\right) \delta 1.49(\mathrm{~s}, 9 \mathrm{H}), 4.12(\mathrm{~d}, J=13.8 \mathrm{~Hz}, 2 \mathrm{H}), 4.25(\mathrm{~d}, J=13.8 \mathrm{~Hz}, 1 \mathrm{H}), 4.91(\mathrm{~d}, J=12 \mathrm{~Hz}, 1 \mathrm{H})$, $5.8(\mathrm{~d}, J=9 \mathrm{~Hz}, 1 \mathrm{H}), 6.22(\mathrm{~d}, J=9 \mathrm{~Hz}, 1 \mathrm{H}), 6.42(\mathrm{sl}, 1 \mathrm{H}), 7.21(\mathrm{t}, J=9 \mathrm{~Hz}, 1 \mathrm{H}), 7.4-7.6(\mathrm{~m}, 11 \mathrm{H})$. Anal. Calcd for $\mathrm{C}_{22} \mathrm{H}_{19} \mathrm{NO}_{2}$ : C, 80.22; H, 5.81; N, 4.25. Found: C, 80.30; H, 5.76; N, 4.18.

6,7-Dihydro-5H-dibenzo[c,e]azepine (S)-2-phenyl-2-hydroxyacetyl amide (2l): Yellow prisms; m.p. $124.6-127.3^{\circ} \mathrm{C} .[\alpha]_{\mathrm{D}}=+92.9\left(c=1.00, \mathrm{CHCl}_{3}\right) ;{ }^{1} \mathrm{H}$ NMR $\left(600 \mathrm{MHz}, \mathrm{CDCl}_{3}\right) \delta 3.95(\mathrm{~d}, 1 \mathrm{H}, J$ $\left.=13.2 \mathrm{~Hz}, \mathrm{NCH}_{2}\right), 4.08\left(\mathrm{~d}, 1 \mathrm{H}, \mathrm{NCH}_{2}\right), 4.19\left(\mathrm{br} \mathrm{d}, 1 \mathrm{H}, \mathrm{NCH}_{2}\right), 4.86(\mathrm{~d}, 1 \mathrm{H}, J=6.5 \mathrm{~Hz}, \mathrm{OH}), 4.91$ (br d, 1H, $\left.\mathrm{NCH}_{2}\right), 5.36(\mathrm{~d}, 1 \mathrm{H}, J=6.5 \mathrm{~Hz}, \mathrm{OCH}), 6.32$ (br d, 1H, Ar), 7.18 (t, J=7.3 Hz, 1H, Ar), 7.40-7.45 (m ,7H, Ar), 7.47-7.51 (m, 4H, Ar); ${ }^{13} \mathrm{C}$ NMR (150 MHz, $\left.\mathrm{CDCl}_{3}\right) \delta 47.33\left(\mathrm{NCH}_{2}\right)$, 47.63 $\left(\mathrm{NCH}_{2}\right), 72.32(\mathrm{OCH}), 127.88(2 \mathrm{CH}, \mathrm{Ph}), 128.10(\mathrm{CH}), 128.16(\mathrm{CH}), 128.23(\mathrm{CH}), 128.53(\mathrm{CH})$, $128.79(\mathrm{CH}), \quad 128.87(\mathrm{CH}), \quad 128.98(\mathrm{CH}), \quad 129.04(\mathrm{CH}), \quad 129.38(2 \mathrm{CH}, \mathrm{Ph}), \quad 130.27(\mathrm{CH}, \mathrm{Ph})$, 132.24(quat), 132.91(quat), 139.61(quat, Ph), 140.51(quat), 140.53(quat), 170.06(quat, NCO). Anal. Calcd for $\mathrm{C}_{27} \mathrm{H}_{28} \mathrm{~N}_{2} \mathrm{O}_{3}$ : C, 75.68; H, 6.59; N, 6.54. Found: C, 75.77; H, 6.63; N, 6.60. 
6,7-Dihydro-5H-dibenzo[c,e]azepine (S)-2-hydroxy-3-phenylpropanoyl amide (2m): Clear oil; $[\alpha]_{\mathrm{D}}=-102.95\left(c=0.8, \mathrm{CHCl}_{3}\right) ;{ }^{1} \mathrm{H} \mathrm{NMR}\left(300 \mathrm{MHz}, \mathrm{CDCl}_{3}\right) \delta 2.98\left(\mathrm{dd}, J^{\prime}=13.8 \mathrm{~Hz}, J^{\prime \prime}=6.7\right.$ $\mathrm{Hz}, 1 \mathrm{H}), 3.9\left(\mathrm{dd}, J^{\prime}=13.8 \mathrm{~Hz}, J^{\prime \prime}=5.2 \mathrm{~Hz}, 1 \mathrm{H}\right), 3.77$ (d, $\left.J=13.02 \mathrm{~Hz}, 1 \mathrm{H}\right), 3.89$ (d, $J=7.9 \mathrm{~Hz}$, $1 \mathrm{H}), 4.25(\mathrm{~d}, J=13.0 \mathrm{~Hz}, 1 \mathrm{H}), 4.7(\mathrm{~d}, J=13.0 \mathrm{~Hz}, 1 \mathrm{H}), 4.83\left(\mathrm{dt}, J^{\prime}=J^{\prime \prime}=6.7 \mathrm{~Hz}, 1 \mathrm{H}\right), 7.2-7.6$ (m, 13H). Anal. Calcd for $\mathrm{C}_{23} \mathrm{H}_{21} \mathrm{NO}_{2}$ : C, 80.44; H, 6.16; N, 4.08. Found: C, 80.48; H, 6.21; N, 4.13.

Table S1. Diastereoisomeric ratios of the biphenyl amides 2, coalescence temperatures, and free energies of activation involved in the biphenyl torsion. ${ }^{a}$

\begin{tabular}{cccc}
\hline Compound & $P: M$ ratio & $\begin{array}{c}T \\
{[\mathrm{~K}]}\end{array}$ & $\begin{array}{c}\Delta \mathrm{G}^{\ddagger} \\
{\left[\mathrm{kcal} \mathrm{mol}^{-1}\right]}\end{array}$ \\
\hline $\mathbf{2 a}$ & $80: 20$ & 213 & 10.15 \\
$\mathbf{2 e}$ & $75: 25$ & 218 & 10.25 \\
$\mathbf{2 h}$ & $95: 5$ & 222 & 11.20 \\
$\mathbf{2 l}$ & $25: 75$ & 223 & 10.40
\end{tabular}

${ }^{a}$ All the activation parameters were measured from either the ${ }^{1} \mathrm{H}$ or ${ }^{13} \mathrm{C}$ NMR spectra. 


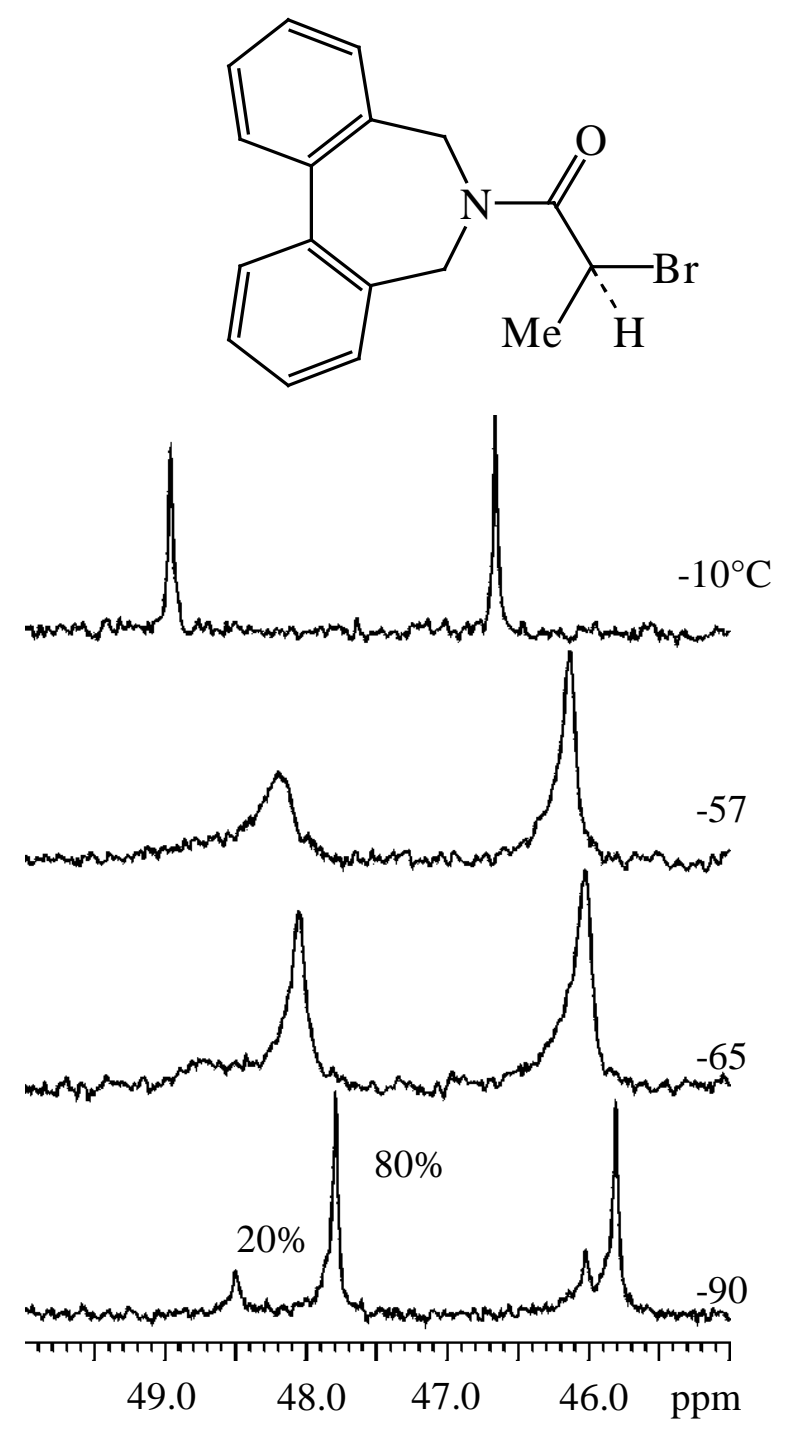

Figure S1. ${ }^{13} \mathrm{C}$ NMR $\left(150 \mathrm{MHz}, \mathrm{CD}_{2} \mathrm{Cl}_{2}\right)$ temperature dependence of the $\mathrm{NCH}_{2}$ signals at 46.6 and 48.9 ppm of $\mathbf{2 a}$. 


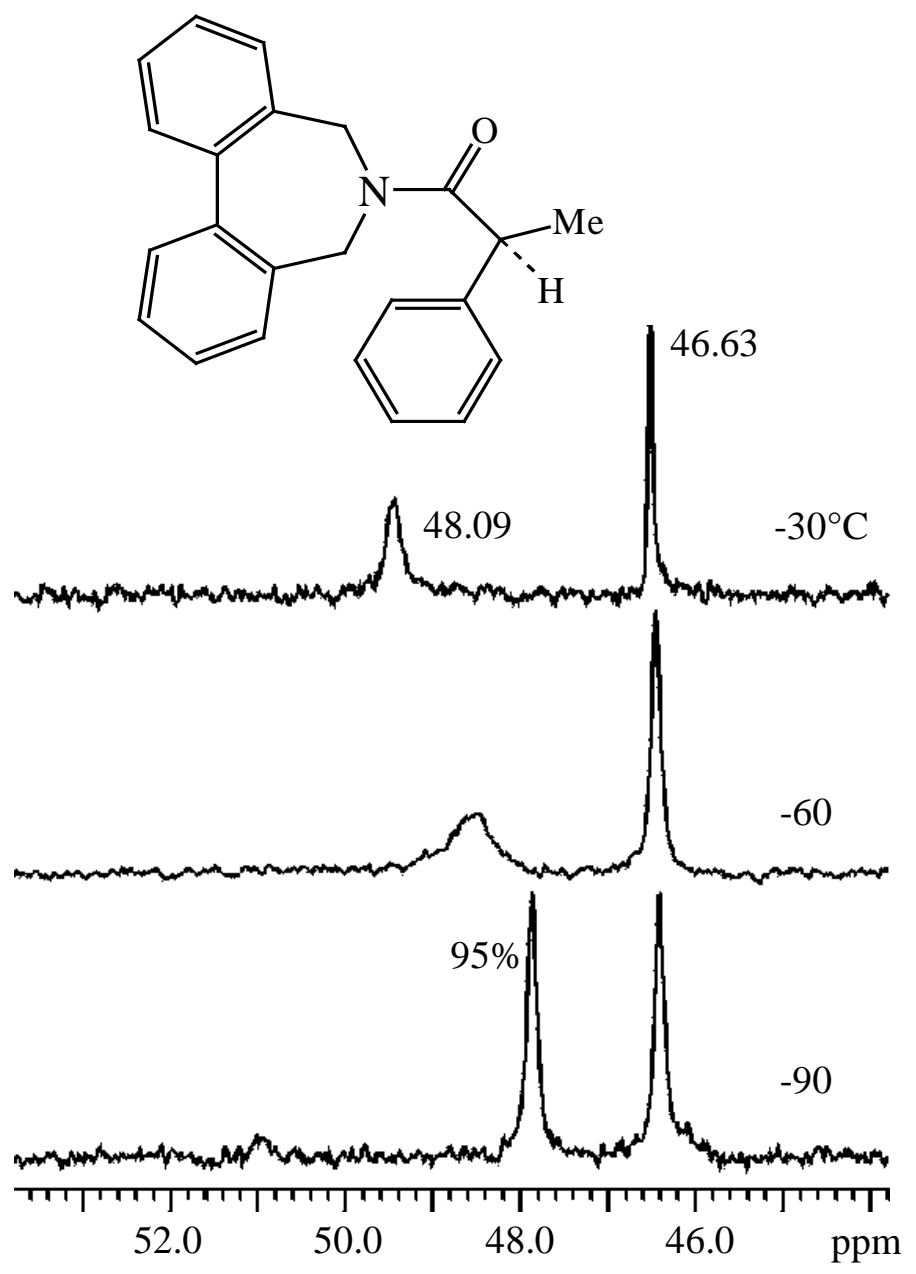

Figure S2. ${ }^{13} \mathrm{C} \mathrm{NMR}\left(150 \mathrm{MHz}, \mathrm{CHF}_{2} \mathrm{Cl}-\mathrm{C}_{6} \mathrm{D}_{6}\right)$ of $\mathbf{2 h}$. The traces show the temperature dependence of $\mathrm{NCH}_{2}$ signals at 45.8 and $47.7 \mathrm{ppm}$. 


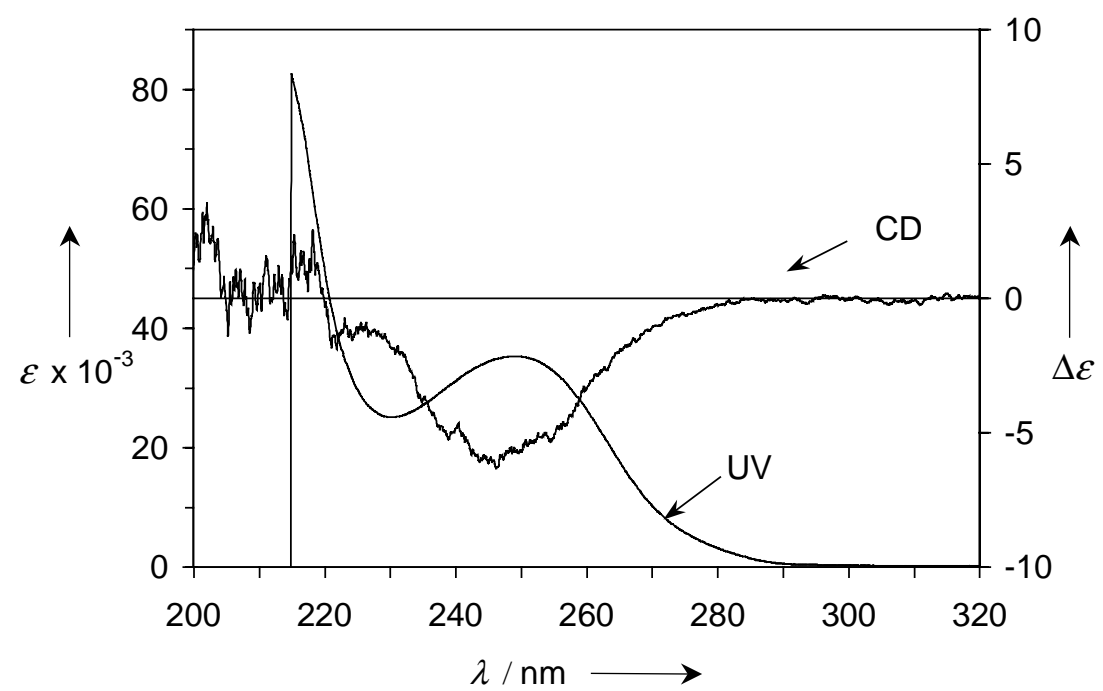

Figure S3. Absorption (UV) and circular dichroism (CD) spectra of $\mathbf{2 b}$ in THF.

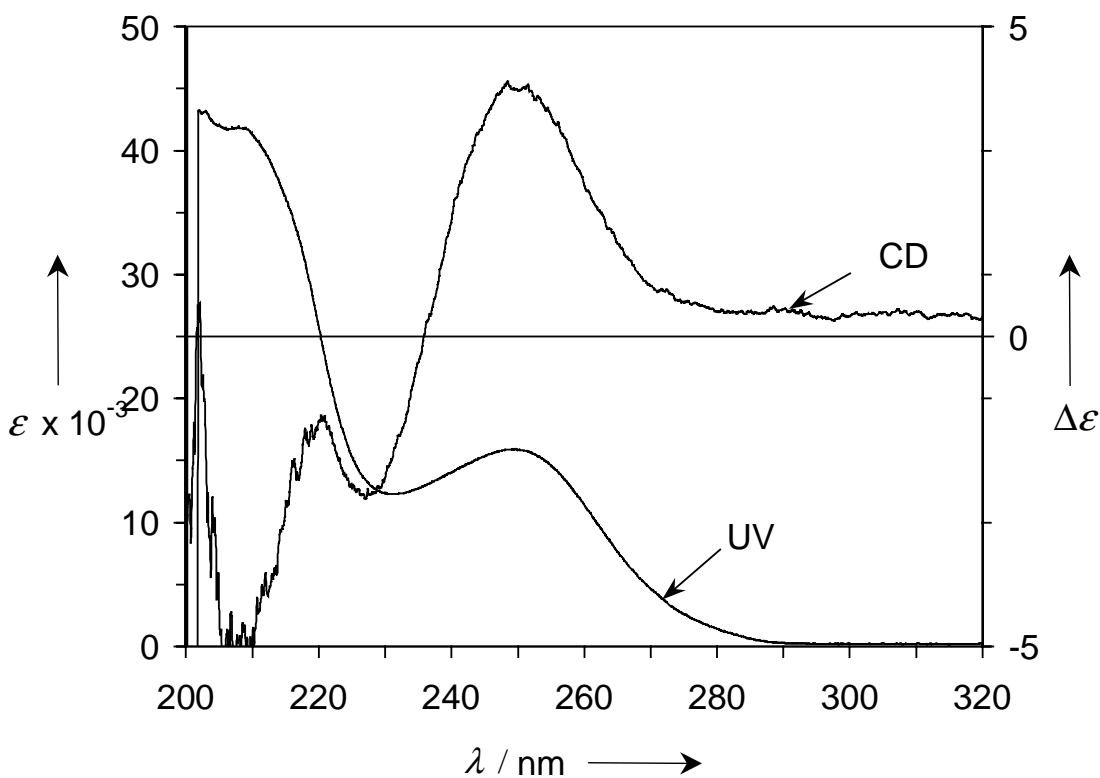

Figure S4. Absorption (UV) and circular dichroism (CD) spectra of $\mathbf{2 c}$ in THF. 


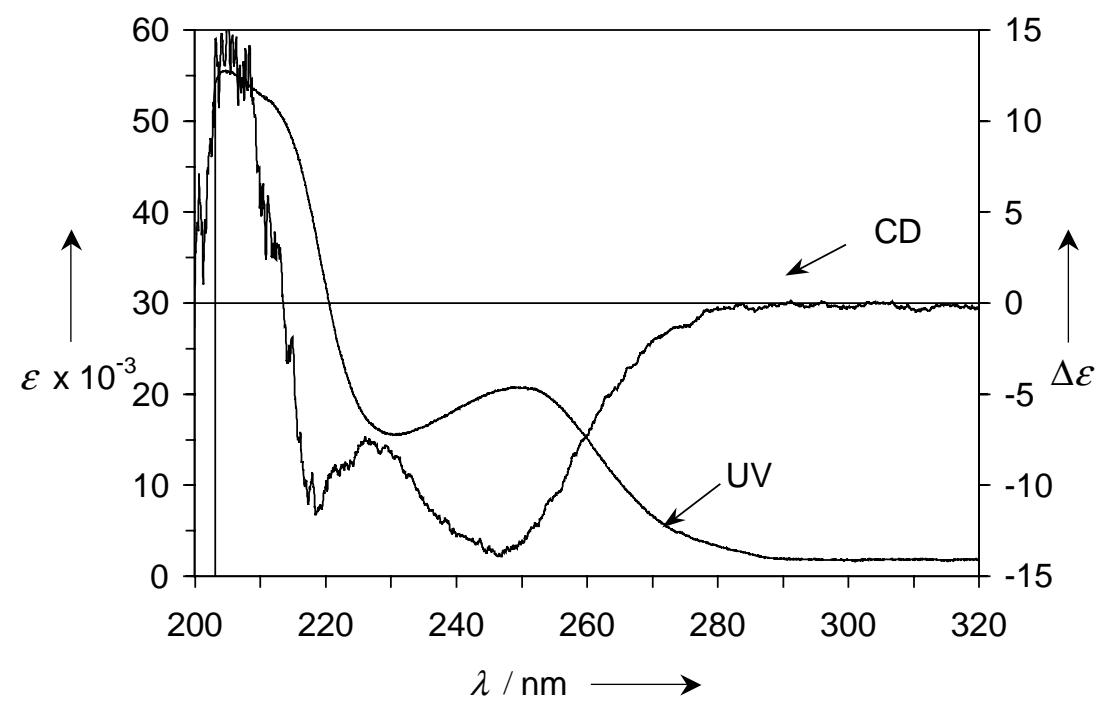

Figure S5. Absorption (UV) and circular dichroism (CD) spectra of $\mathbf{2 d}$ in THF.

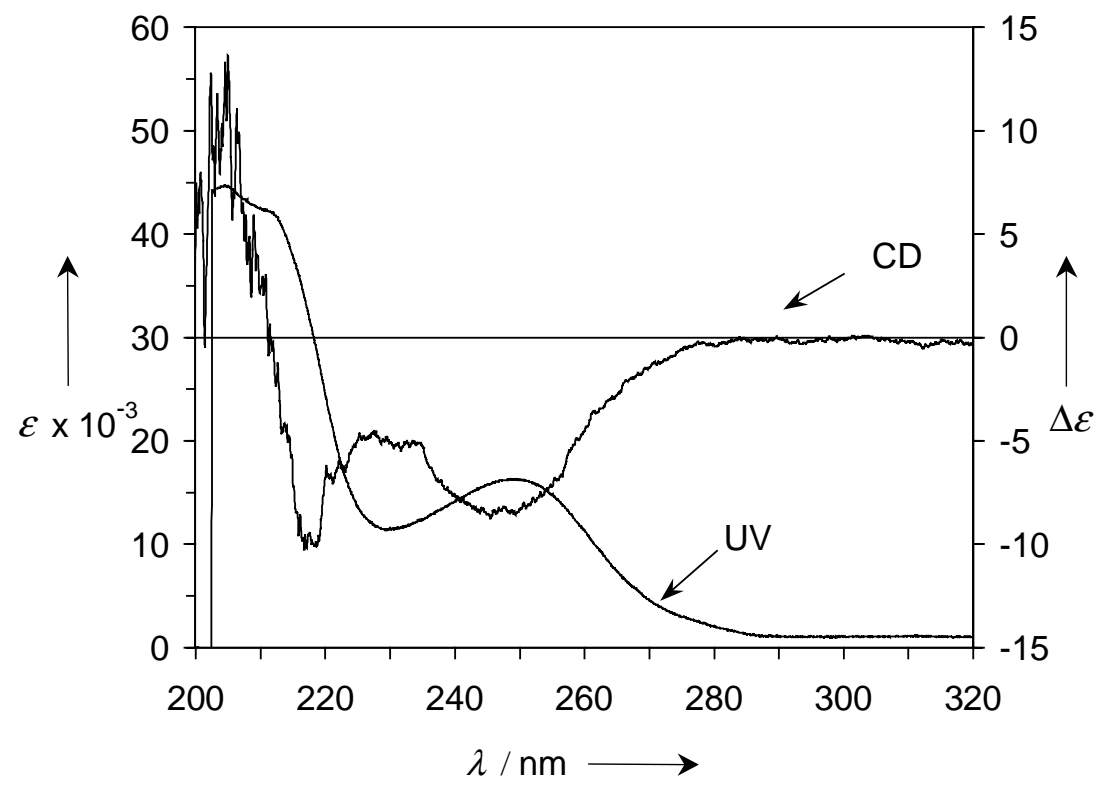

Figure S6. Absorption (UV) and circular dichroism (CD) spectra of $2 \mathbf{e}$ in THF. 


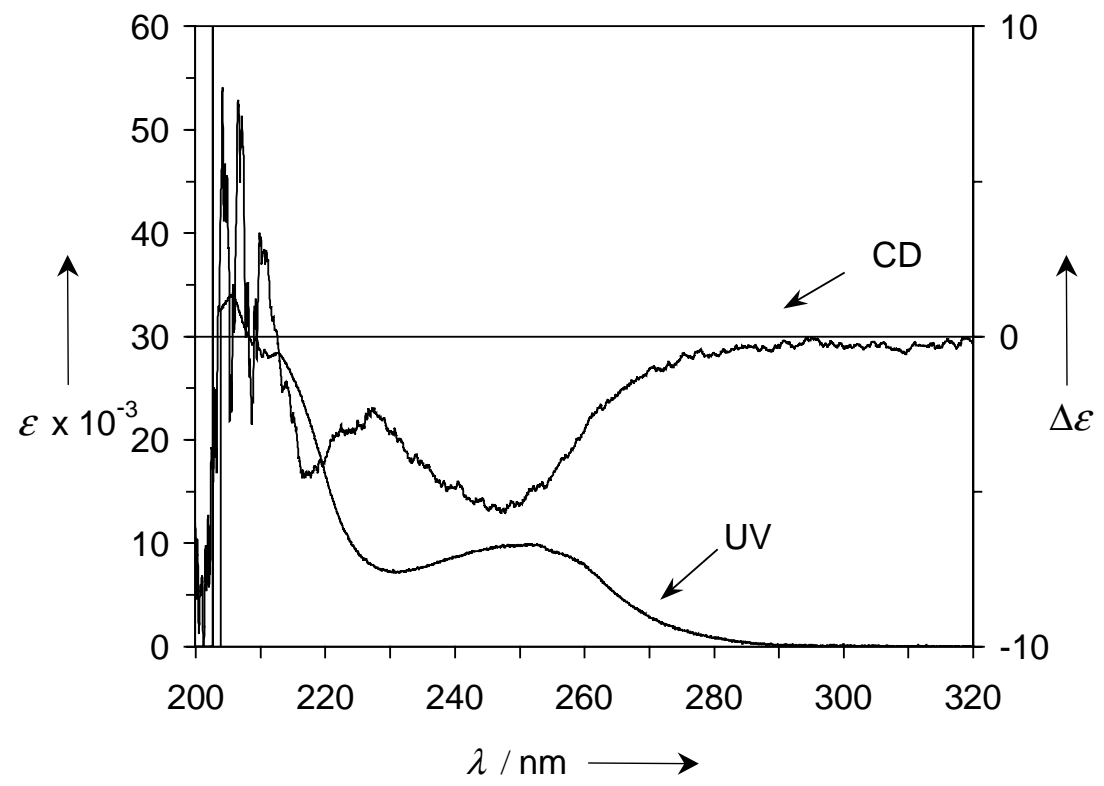

Figure S7. Absorption (UV) and circular dichroism (CD) spectra of $\mathbf{2 f}$ in THF.

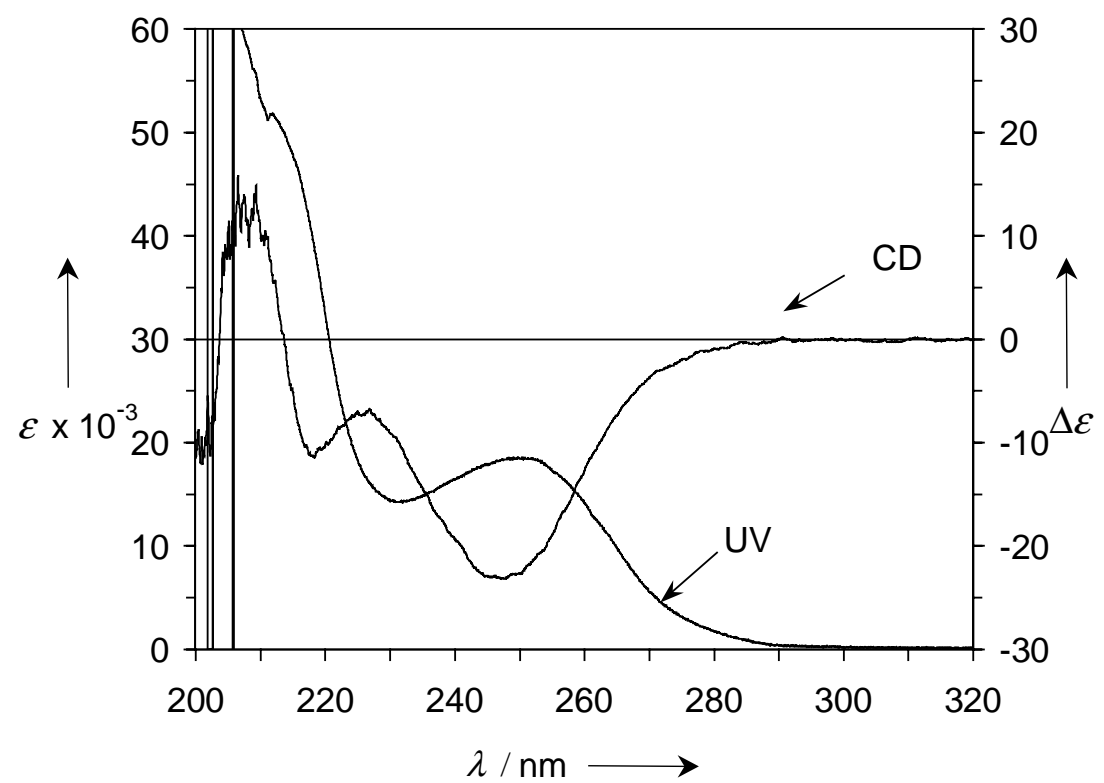

Figure S8. Absorption (UV) and circular dichroism (CD) spectra of $\mathbf{2 g}$ in THF. 


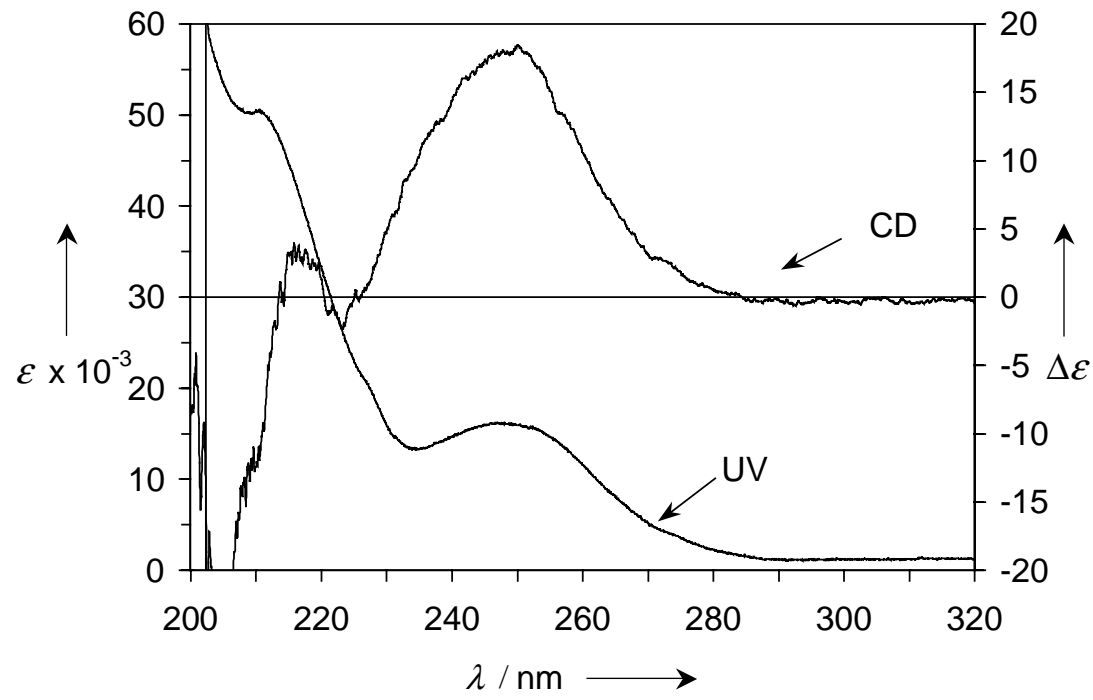

Figure S9. Absorption (UV) and circular dichroism (CD) spectra of $2 \mathbf{i}$ in THF.

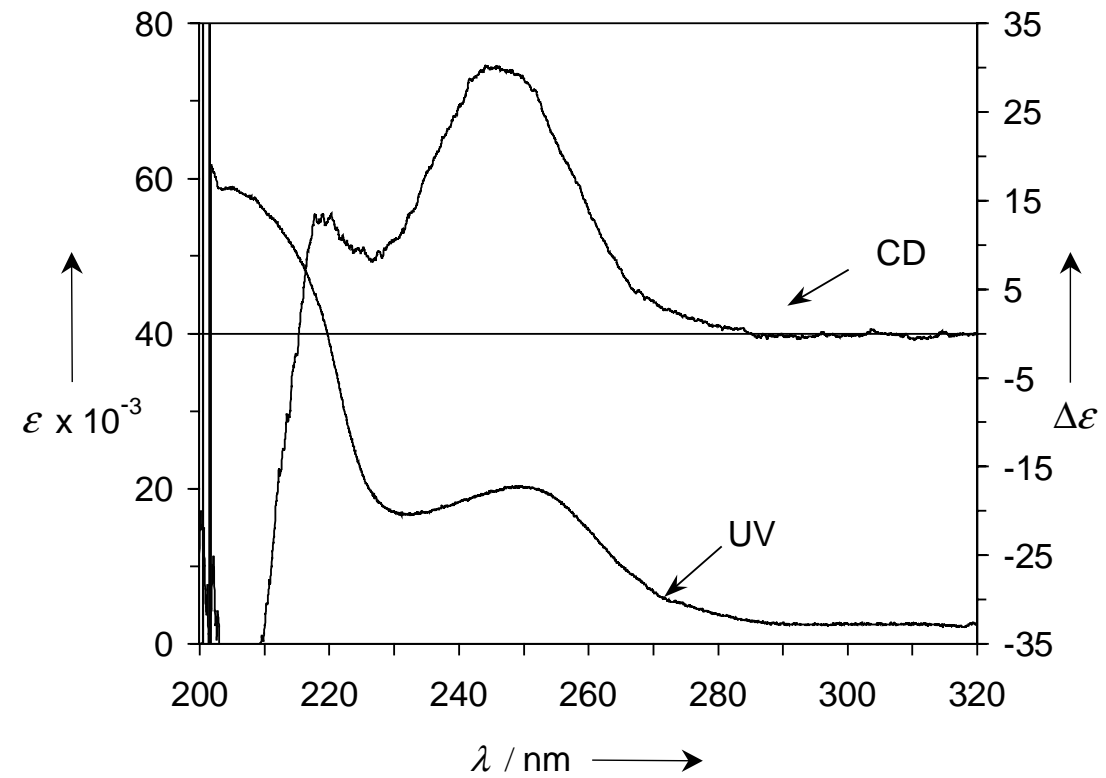

Figure S10. Absorption (UV) and circular dichroism (CD) spectra of $\mathbf{2 j}$ in THF. 


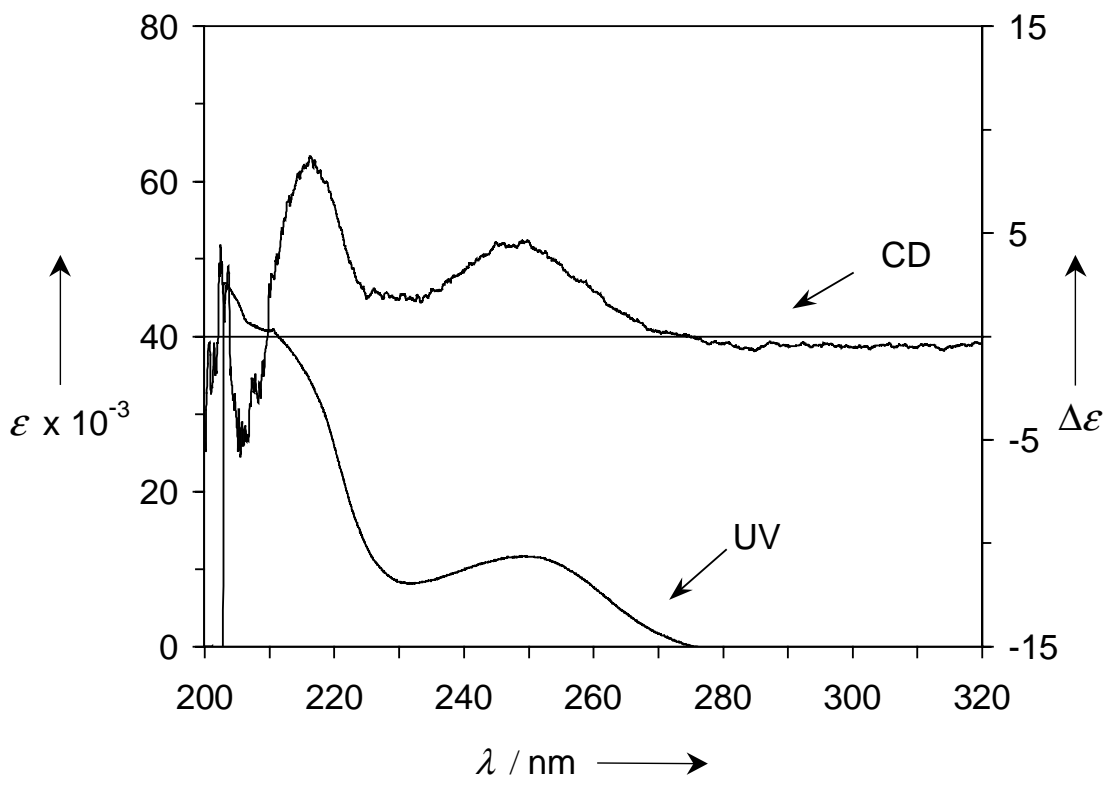

Figure S11. Absorption (UV) and circular dichroism (CD) spectra of $\mathbf{2 k}$ in THF.

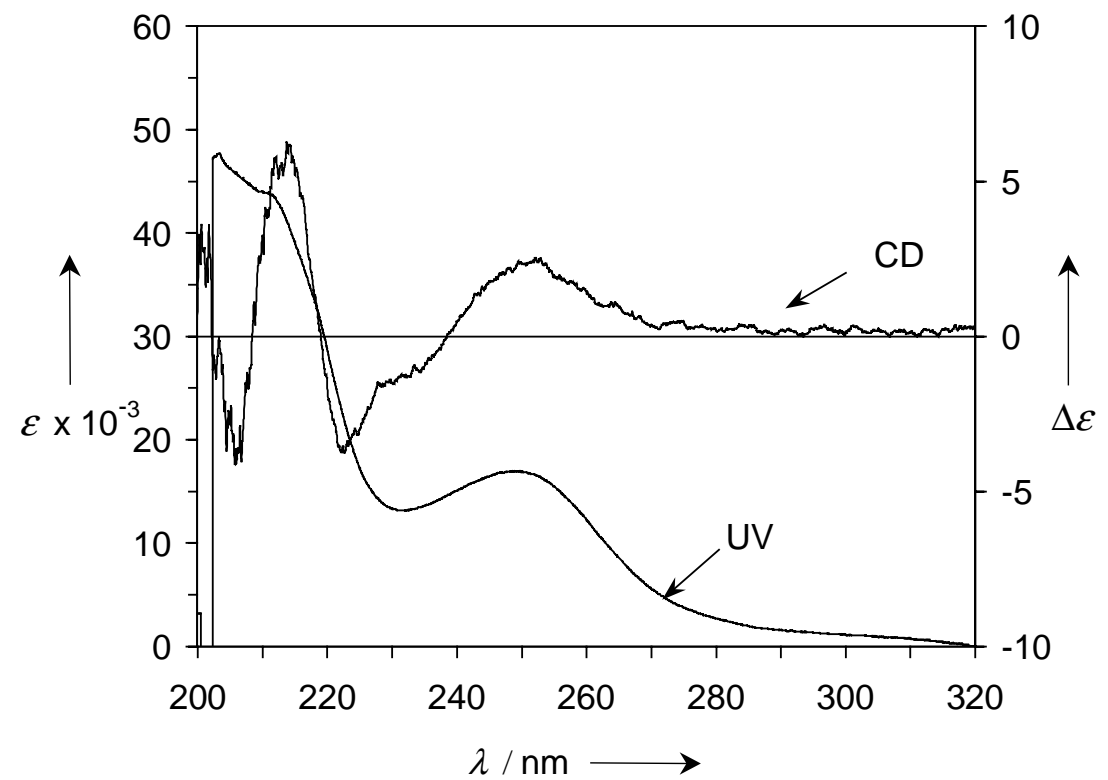

Figure S12. Absorption (UV) and circular dichroism (CD) spectra of $\mathbf{2 l}$ in THF. 


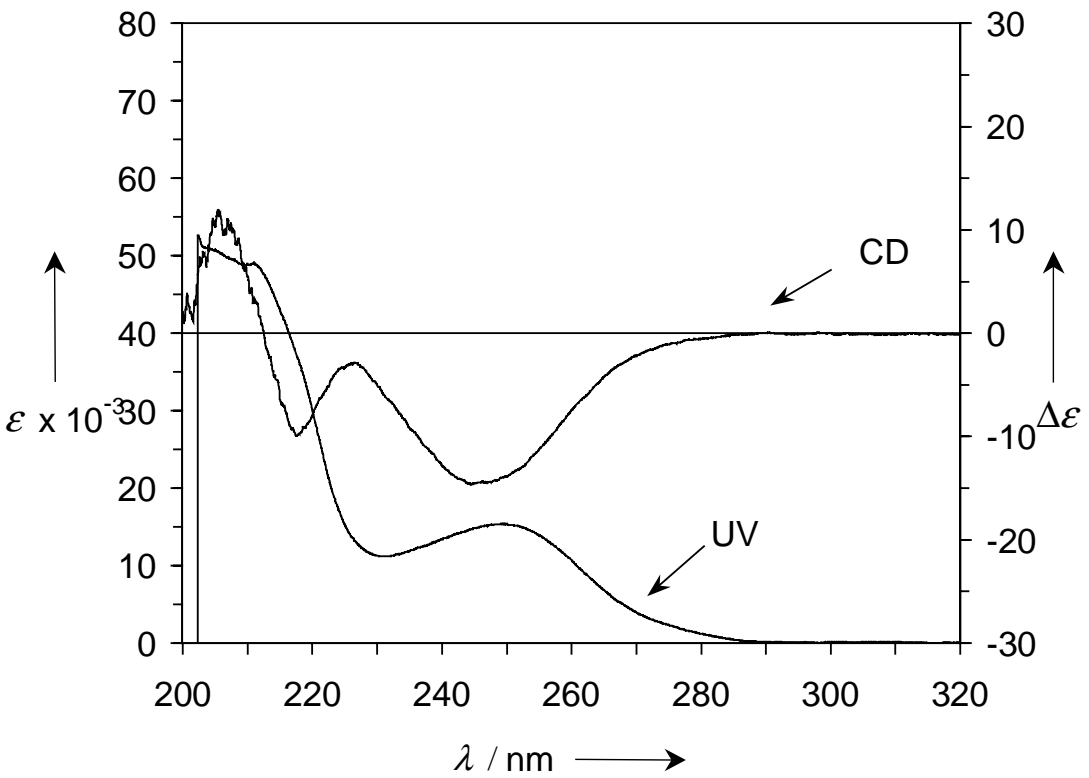

Figure S13. Absorption (UV) and circular dichroism (CD) spectra of $\mathbf{2 m}$ in THF . 